\title{
Electron Holography and Imaging Capabilities of the JEOL 2100F-LM
}

\author{
M. A. Schofield, M. Beleggia, and Y. Zhu \\ Center for Functional Nanomaterials, Brookhaven National Laboratory, Upton NY 11973-5000
}

As the characteristic size of technologies utilizing magnetic phenomena continues to decrease, the need to carry out quantitative magnetic materials research with ever-increasing spatial resolution and sensitivity becomes vital. Of the available techniques to carry out magnetization measurement, offaxis electron holography has significant potential with regard to spatial resolution and sensitivity [1]. By recovering the phase shift of electrons passing through the sample, a direct relation to the electrostatic and magnetostatic potentials associated with the sample is obtained. While electron holography is a compelling technique, the greatest challenges in materials science demand complementary techniques and capabilities. To address these needs, JEOL Inc. has developed a $200 \mathrm{kV}$ field-emission TEM with a long focal-length objective lens. The microscope is equipped with a biprism situated in the selected area aperture plane of the microscope. Design of the objective lens is such that the sample sits in low-field conditions under all operating modes of the microscope. The available magnification range is nominally $50 \mathrm{X}$ to $200 \mathrm{kX}$, and diffraction mode is available to record selected area diffraction patterns. With an $8 \mathrm{~mm}$ pole-piece gap, the microscope can accommodate a variety of stages to perform heating, cooling, and in situ magnetization experiments. We present here measurement of the electron holography and imaging capabilities of this new microscope, along with results illustrating the potential for electron holography studies [2].

Figure 1 summarizes the holography performance of the JEOL 2100F-LM. The fringe spacing, hologram width, and contrast were measured as a function of biprism voltage in the HOLO-M mode of the microscope. With forthcoming improvements to the room environment, and optimization of the lens program, a modest improvement to the holography capabilities can be expected. Nevertheless, Fig.1(a) and (c) show that fringe spacing less than $1.5 \mathrm{~nm}$ can be achieved with a moderate contrast around $10-15 \%$. Alternatively, a biprism voltage around $70-75 \mathrm{~V}$ provides fringe spacing around $1.7 \mathrm{~nm}$. Under these conditions, $5 \mathrm{~nm}$ spatial resolution can easily be obtained with roughly $500 \mathrm{~nm}$ field-of-view. The fringe contrast in this case is $20-25 \%$, which provides generally suitable phase sensitivity for most applications. The spherical aberration coefficient $\mathrm{C}_{\mathrm{s}}$ and defocus is required for holography applications involving high resolution, and are also important when utilizing through-focus techniques to obtain quantitative information complementary to electron holography. A focal series of images of holey carbon were recorded and, following well-documented methods [3], $C_{s}$ and the absolute focus for each image were obtained. Figure 2(a) shows the results where a value of $C_{s}=108.7 \pm 2.2 \mathrm{~mm}$ was found, which compares reasonably with the value of $95 \mathrm{~mm}$ cited by the manufacturer. Figure 2(b) shows results of the absolute focus, which was found to be linear over the range of $\pm 3 \mu \mathrm{m}$ having a minimum focal step $\Delta \mathrm{f}=87.6 \pm 1.4 \mathrm{~nm}$. For quantitative Lorentz imaging, these characteristics of the focal behavior are quite satisfactory, and the measured value of $\mathrm{C}_{\mathrm{s}}$ gives a point resolution of $0.77 \mathrm{~nm}$. In terms of holography applications, this does not impose any limitations since the minimum fringe spacing that can be achieved only approaches $1 \mathrm{~nm}$ and, therefore, $>3 \mathrm{~nm}$ resolution in the reconstructed wave.

Figure 3 shows holography results from a 94nm latex sphere dispersed on holey carbon substrate. The cosine map of the reconstructed phase (Fig.3a) is amplified 16x to show the fringing field associated with the charged sphere. The phase was reconstructed from hologram recorded with $1.97 \mathrm{~nm}$ interference fringes and $18 \%$ contrast in the sample. A profile of the circularly averaged phase shift across the sphere is shown in Fig.3(b), along with the calculated phase shift shown as a red line. The best-fit parameters of the calculated phase shift gives a mean inner potential of $5.4 \mathrm{~V}$ and 65 electrons of charge distributed on the surface of the latex sphere. While the principle utility of the JEOL $2100 \mathrm{~F}$ is for application to magnetic studies, these results illustrate the comparatively high resolution and sensitivity of the holography capabilities of this microscope. Additional results and applications involving magnetic materials will be presented at the conference [4]. 


\section{References}

[1] E. Völkl et al., Eds., Introduction to Electron Holography, Plenum, New York, 1999.

[2] M. A. Schofield, M. Beleggia and Y. Zhu, submitted to Ultramicroscopy, 2005.

[3] P. Busek et al., Eds., High-Resolution Transmission Electron Microscopy and Associated Techniques, Oxford University Press, New York, 1992.

[4] Supported by US Department of Energy BES, under contract No. DE-AC02-98CH10886. We are grateful for valuable discussion and assistance kindly provided by Dr G. Matteucci.
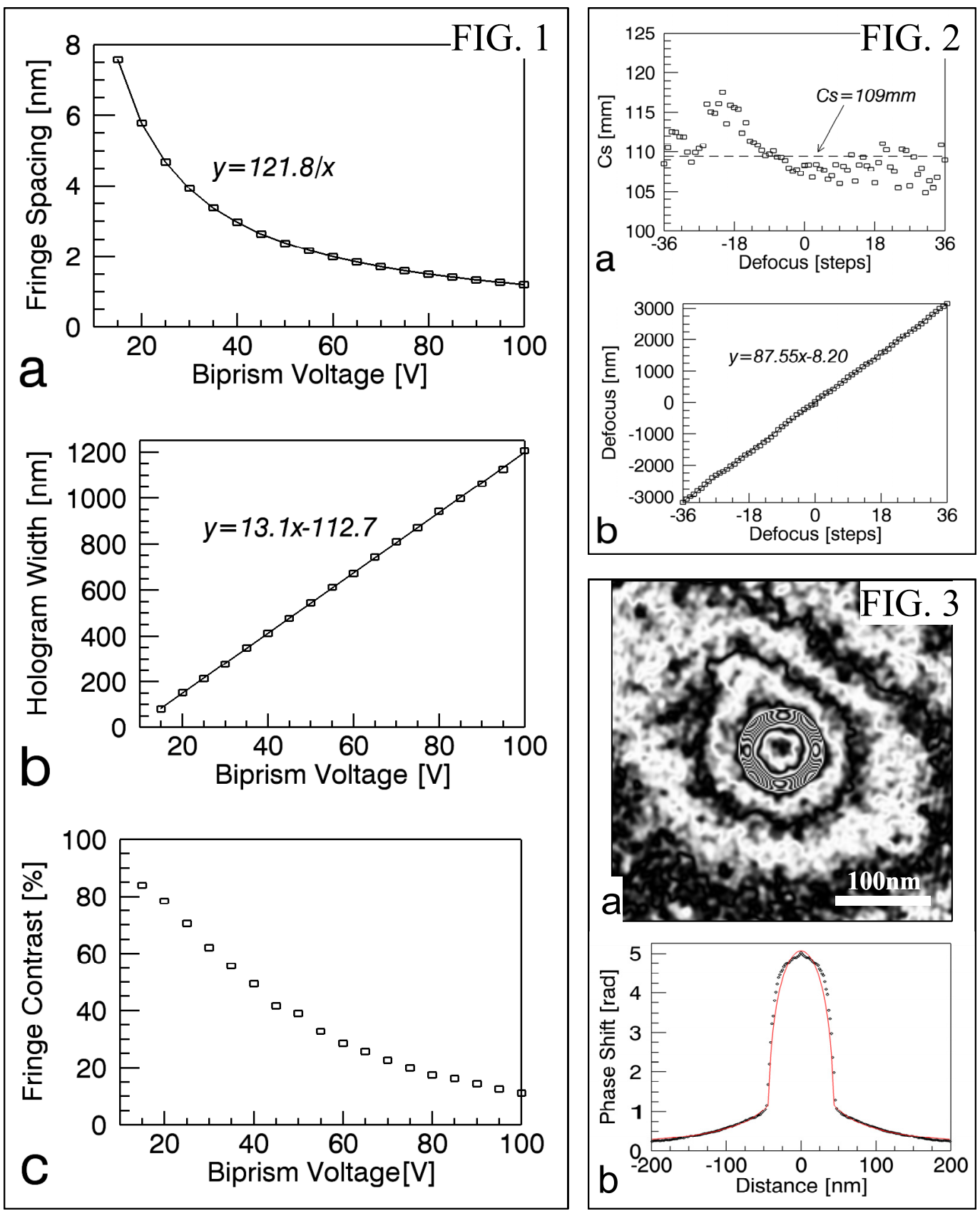

FIG. 1. (a) fringe spacing, (b) hologram width, and (c) fringe contrast as a function of biprism bias. FIG. 2. (a) coefficient of spherical aberration, and (b) image defocus as a function of focal steps.

FIG. 3. (a) cosine of 16x amplified reconstructed phase from latex sphere, and (b) calculated phase shift (red line) for a sphere with 5.4V inner potential and 65 electrons distributed on its surface. 